\title{
Immunoreactivity of arcuate nucleus astrocytes in rats after intragastric administration of habanero peppers (Capsicum Chinese Jacq.)
}

\author{
K. Rycerz ${ }^{1}$, A. Krawczyk ${ }^{1}$, J. Jaworska-Adamu1, M. Gołyński², \\ K. Lutnicki², I. Balicki ${ }^{3}$ \\ ${ }^{1}$ Department of Animal Anatomy and Histology, Faculty of Veterinary Medicine, \\ University of Life Sciences, Akademicka 12, 20-033, Lublin, Poland \\ ${ }^{2}$ Subdepartment of Internal Diseases of Farm Animals and Horses, Faculty of Veterinary Medicine, \\ University of Life Sciences, Głęboka 30, 20-612 Lublin, Poland \\ ${ }^{3}$ Department and Clinic of Animal Surgery, Faculty of Veterinary Medicine, \\ University of Life Sciences, Głęboka 30, 20-612 Lublin, Poland
}

\begin{abstract}
Habanero pepper fruits contain capsaicin (CAP) characterised by a spicy taste. Astrocytes express vanilloid receptor (TRPV1), which interacts with cannabinoids including CAP. Only a few studies revealed that CAP leads to alterations of the arcuate nucleus (ARC) structures. The aim of this study was to analyse the GFAP (GFAP-IR) and S100ß (S100ß-IR) immunoreactive astrocytes of ARC in adult rats after intragastric administration of habanero pepper fruits. Adult, Wistar rats received a peanut oil - control group (C) - and oil suspension of habanero pepper fruits at a dose of $0.08 \mathrm{~g} \mathrm{dm} / \mathrm{kg}$ b.w. for 7 days - E1 group - and 28 days - E2 group. After euthanasia, the brains were embedded in paraffin blocks using a routine histological technique. Frontal slices of ARC were immunohistochemically stained for GFAP and S100 $\beta$ using specific antibodies in the peroxidase-antiperoxidase method. Astrocytes of ARC were morphologically and morphometrically analysed under a light microscope. The results of the study did not reveal statistically significant changes in the density of GFAP-IR cells in E1 and E2 groups of rats in comparison with group C. A statistically significant increase in the density of S100ß-IR astrocytes was observed in the E1 group and a decrease in the E2 group. Astrocytes with expression of both studied proteins were characterised by morphological alterations in ARC in the E2 group. The obtained results suggest an influence of CAP contained in habanero pepper fruits on the reactivity of astroglia, which may have an impact on the astrocyte-neuron interactions in order to maintain a proper activity of nervous cells in ARC.
\end{abstract}

Key words: capsaicin, astrocytes, GFAP, S100ß, arcuate nucleus, rats 


\section{Introduction}

Habanero pepper fruits (Capsicum chinense Jacq.) contain an active alkaloid called capsaicin (CAP) which is characterised by a spicy and burning taste. These fruits are used in the food, pharmaceutical and cosmetic industries. The most important properties of CAP are the irritant and analgesic effect, and influence on fat tissue metabolism and thermoregulation. The influence of CAP on the organism depends on the dose, timing and the route of administration (Pieńko 2013). Intragastric application of CAP at a high dose to rats $(50 \mathrm{mg} / \mathrm{kg}$ b.w. for 60 days) has a negligible impact, leading only to the inhibition of growth. (Monsereenusorn 1983). This alkaloid is mildly toxic after oral administration. Hydrolysing enzymes take part in its metabolism which occurs in the gastrointestinal tract (Surh and Lee 1995). However, less than $5 \%$ of an unchanged form of CAP enters the human and rat brain with blood (O'Neill et al. 2012). CAP in low doses exhibits medical properties but the severe toxic effect of high doses of the alkaloid cannot be excluded (Olszewska 2010). Its neurotoxic effect was described in rats after systematic administration (Sugimoto et al. 1998). Moreover, a neurotoxic effect of CAP was demonstrated in adult humans after subcutaneous administration. (Simone et al. 1998). CAP may be transported via the blood-brain barrier (BBB), although the mechanism of the transport is not known (Güler et al. 2012).

The arcuate nucleus (ARC) of the hypothalamus is involved, inter alia, in the regulation of the food intake mechanism and body weight control. ARC, via numerous connections with other areas of the brain, takes part in many functions. It is involved in the regulation of secretory mechanisms of the pituitary gland, in the neuroendocrine integration of the organism, in the reproduction, in the response to stress and the control of pain (Belousov and van den Pol 1997). ARC neurons produce numerous neurotransmitters including glutamate and modulators such as neuropeptide Y (NPY) and cholecystokinin (CCK) (Park et al. 2004).

CAP binds to a vanilloid membrane TRPV1 receptor (transient receptor potential vanilloid subtype 1) also known as capsaicin receptor in the hypothalamic structures (O'Neil and Brown 2003). Not only the nerve cells but also the astrocytes have a TRPV1 mRNA and the protein that can interact with the cannabinoids, including CAP (Toth et al. 2005).

Glial fibrillary acidic protein (GFAP) and S100 $\beta$ protein are the markers of mature astrocytes. GFAP establishes the glial intermediate filaments (IFs) forming the cell cytoskeleton (Montgomery 1994, Eng et al. 2000). A large expression of GFAP was demonstrated in astrocytes of ARC. However, it may dynamically change depending on the microenvironment conditions. Under the influence of many factors, astrocytes may be morphologically altered, including the reorganization of the filaments and functional changes, which affect the neurons (Tardy et al. 1993, Eng et al. 2000).

S100 $\beta$, which belongs to calcium binding proteins from the EF-hand family, is localised in the nuclei and cytoplasm of astrocytes. This protein occurs as free or it binds to membranes, type III intermediate filaments, centrioles, microtubules and the mitotic spindle. It decreases calcium concentration in cells and thus it acts as a buffer. After releasing of the protein to intracellular space by astrocytes it affects the neurons and other glia. S100 $\beta$ in nanomolar concentration has a trophic effect on nervous system structures (CNS) and in micromolar concentration it is toxic and leads to apoptosis of neurons and glia (Donato 2003).

Only a few studies demonstrated the effect of CAP on brain structures depending on the route, dose and time of administration. A significant increase of GFAP and glutamine synthetase immunoreactivity in ARC and in median eminence (ME) was demonstrated in rats after subcutaneous administration of CAP (Okere and Waterhouse 2004). CAP effect on cholecystokinin (CCK) and neuropeptide Y (NPY) expression was described in some areas of the brain in rats after oral administration using a fat rich diet (Park et al 2004).

Untill now, the impact of CAP on astrocytes after intragastric administration of habanero pepper has not been shown. Therefore, the aim of this study was to morphologically and morphometrically analyse the GFAP (GFAP-IR) and the S100 $\beta$ (S100 $\beta$-IR) immunoreactive astrocytes of ARC in adult rats after 8-day and 4-week administration of habanero pepper fruits.

\section{Materials and Methods}

\section{Animals}

The experiments were approved by the Second Local Ethics Committee in Lublin (No. 21/2013). The study was conducted on 15 adult male Wistar rats (120-125 g) from a laboratory animal farm. The animals were kept in an air-conditioned room with relative air humidity of $45-47 \%$ and a temperature of $22-23^{\circ} \mathrm{C}$ in a $12 \mathrm{~h}$ light $/ 12 \mathrm{~h}$ dark cycle. The rats were fed with commercial fodder for laboratory animals (LSM, Agropol Motycz Poland) and they had perma- 
nent access to water ad libitum. The acclimation period was 16 days prior to the experiment. The rats $(n=15)$ were divided into two experimental (E1 and E2) and one control group (C). Field, dry fruits of habanero pepper with HPCR - the specific content of capsaicin of $7.64 \mathrm{mg} / \mathrm{dm}$ (capsaicin and dihydrocapsaicin) were suspended in peanut oil. The suspension was administered to the rats at a dose of $0.08 \mathrm{~g} \mathrm{dm} / \mathrm{kg}$ b.w. for a period of 7 days (E1) and 28 days (E2). Daily doses were divided into 2 equal parts and administrated every $12 \mathrm{~h}$. The suspension concentrations (about $0.5 \mathrm{ml}$ ) were prepared for every animal and was applied intragastrically using special, automatic plastic tubes for feeding of rodents (Instech Laboratories, Inc., Plymouth Meeting, PA USA). Control animals (C) received approximately $0.5 \mathrm{ml}$ of pure peanut oil using the same method. The rats were euthanised $12 \mathrm{~h}$ after the last administration of the suspension. The brains were immediately dissected, fixed in fresh and buffered $10 \%$ formalin (pH-7.0) for 12 $\mathrm{h}$ at $4^{\circ} \mathrm{C}$ and embedded in paraffin blocks using a routine histological technique. Frontal, $6 \mu \mathrm{m}$-thick slices containing ARC were obtained in the microtome.

\section{Immunohistochemistry}

To inhibit the endogenous peroxidase activity, brain slices containing ARC were treated with $0.4 \%$ $\mathrm{H}_{2} \mathrm{O}_{2}$ for $30 \mathrm{~min}$ at room temperature (rt). In order to eliminate background staining the slides were incubated in 10\% goat serum (Sigma-Aldrich) for $20 \mathrm{~min}$. Immunohistochemical detection of astrocytic markers GFAP and S100 $\beta$ was carried out using a peroxidase-antiperoxidase method (PAP). A set of antibodies (Sigma-Aldrich) and reagents diluted in $0.5 \mathrm{M}$ Tris buffer (TBS, pH-7.6) were used. The monoclonal rabbit anti-GFAP antibody and mouse anti-S100ß antibody were applied on the slides to reveal astrocytic proteins. The material was incubated in primary antibodies for $24 \mathrm{~h}$ at $4^{\circ} \mathrm{C}$. Subsequently, the secondary, species appropriate, monoclonal anti-IgG-peroxidase antibody was used for $1 \mathrm{~h}$ at $\mathrm{rt}$. The dilutions of antibodies were prepared according to the producer's recommendations. Diaminobenzidine (DAB) was used as a chromogen. Additionally, after immunostaining, the slides were counterstained with Mayer's haematoxylin. A reaction specificity control was performed omitting the primary antibodies and replacing them with normal goat serum. GFAP-IR and S100 $\beta$-IR in ARC were analysed and photographed under a light microscope BX51 (Tokyo, Japan) using an Olympus Color View IIIu digital camera. On the basis of GFAP-IR and S100 $\beta$-IR the morphology of the astrocytes was analysed.

\section{Morphometric analyses}

Average density of GFAP and S100 $\beta$-immunopositive cells in $\mathrm{ARC}$ was analysed in the $\mathrm{Cell}^{\wedge} \mathrm{D}$ program (Olympus, Tokyo, Japan). From every group $(\mathrm{C}, \mathrm{E} 1, \mathrm{E} 2) 25$ slices were randomly chosen for each studied protein. Two photos were taken from each slice. A grid of squares $150 \times 150 \mu \mathrm{m}\left(22.5 \times 10^{-3} \mathrm{~mm}^{2}\right)$ was imposed on the microphotographs. GFAP-IR and S100ß-IR astrocytes were counted in 2 randomly selected squares from each photo. Measurements of 100 squares were obtained per each studied group for both proteins. Statistical analyses were carried out with the $\mathrm{R}$ 3.0.2 program. In order to compare the results a nonparametric Kruskal-Wallis test was used. The significance factor was set to 0.05 .

\section{Results}

\section{Microscopic analyses of GFAP-IR astrocytes in ARC}

Immunohistochemical reactions revealed the presence of astrocytes with GFAP expression in ARC in all studied groups. The protein was localised in the cytoplasm of cell bodies and processes.

Numerous astrocytes with intensive GFAP-IR were present in rats' ARC of C and E1 groups. Many primary and thick processes arose from the cell bodies and divided into secondary, thinner branches. Oval or round nuclei were localised in the centre of the cytoplasm. Astrocytes with fewer processes were observed very seldom (Fig. 1, 2). On the other hand, in ARC of animals from the E2 group the astrocytes were morphologically altered. In their oval cell bodies the nuclei were localised eccentrically and a few, thick primary processes divided into short branches arose from the other side of the cell (Fig. 3).

\section{Microscopic analyses of the S100 $\beta$-IR astrocytes in ARC}

Immunohistochemical reactions demonstrated the presence of S100 $\beta$-positive astrocytes in ARC in all groups of animals. In the analysed area of the brain, in rats from $\mathrm{C}$ and $\mathrm{E} 1$ groups a reaction product was localised mainly in the nuclei and cytoplasm of cell bodies. Initial parts of the processes were seldom observed (Fig. 4, 5). However, oval cells with two or three thick processes divided into minor branches were present in rats' ARC from the E2 group (Fig. 6). Moreover, an astroglia with similar morphology as in animals from the $\mathrm{C}$ and $\mathrm{E} 1$ groups was sparsely observed. 


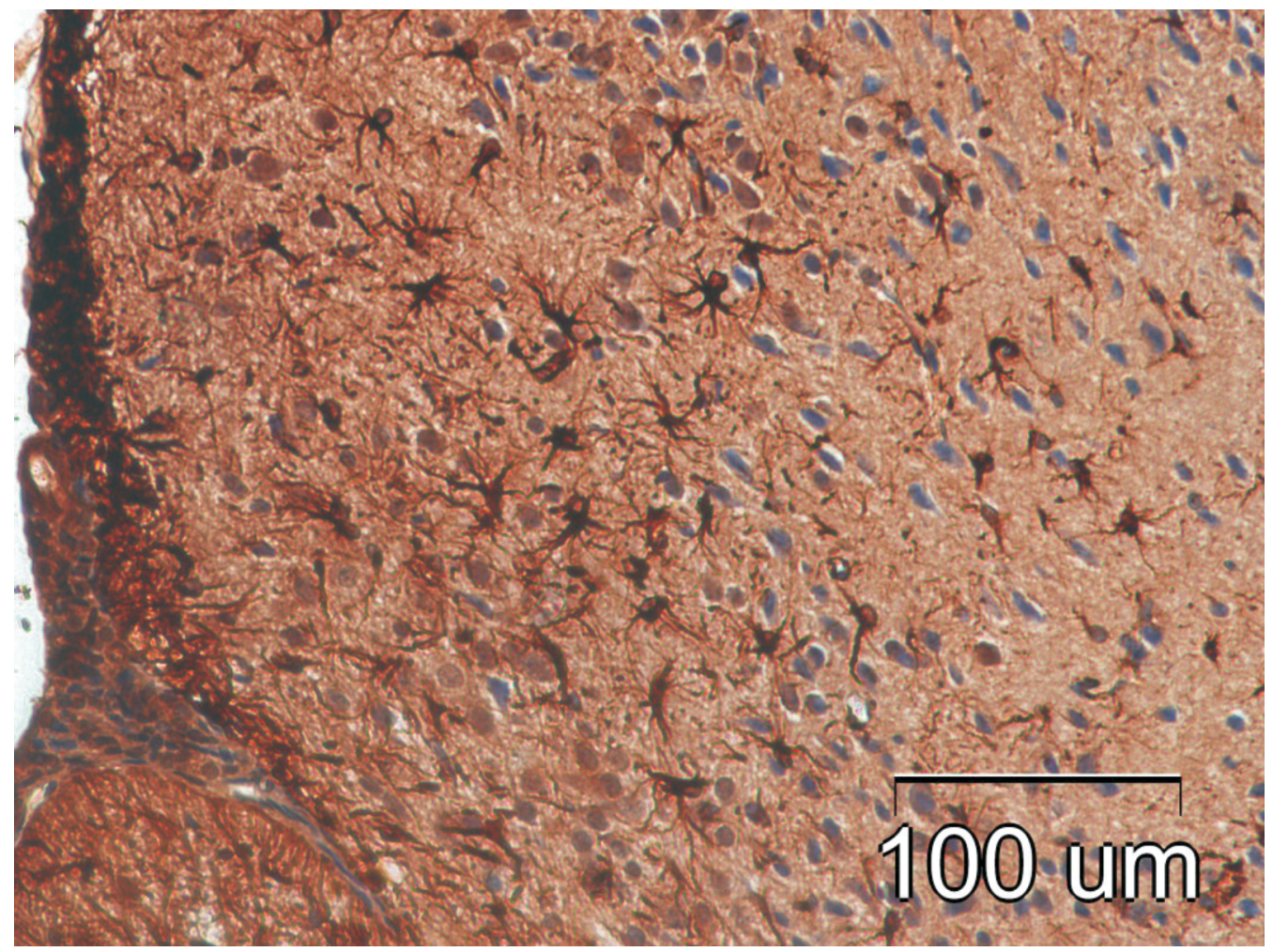

Fig. 1. Immunoreactivity of the GFAP-positive astrocytes in the arcuate nucleus (ARC) of C group.

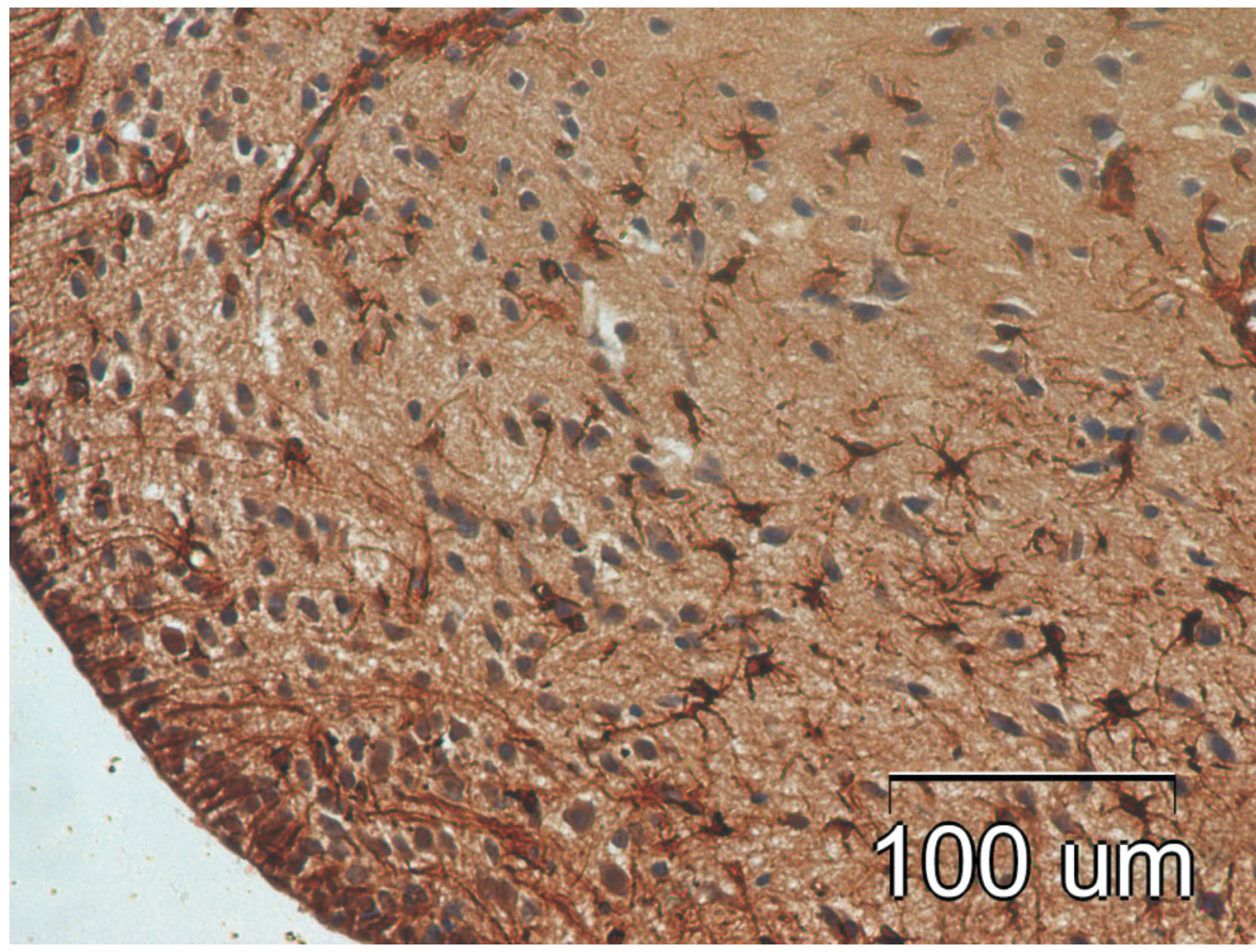

Fig. 2. Immunoreactivity of the GFAP-positive astrocytes in the ARC of E1 group. 


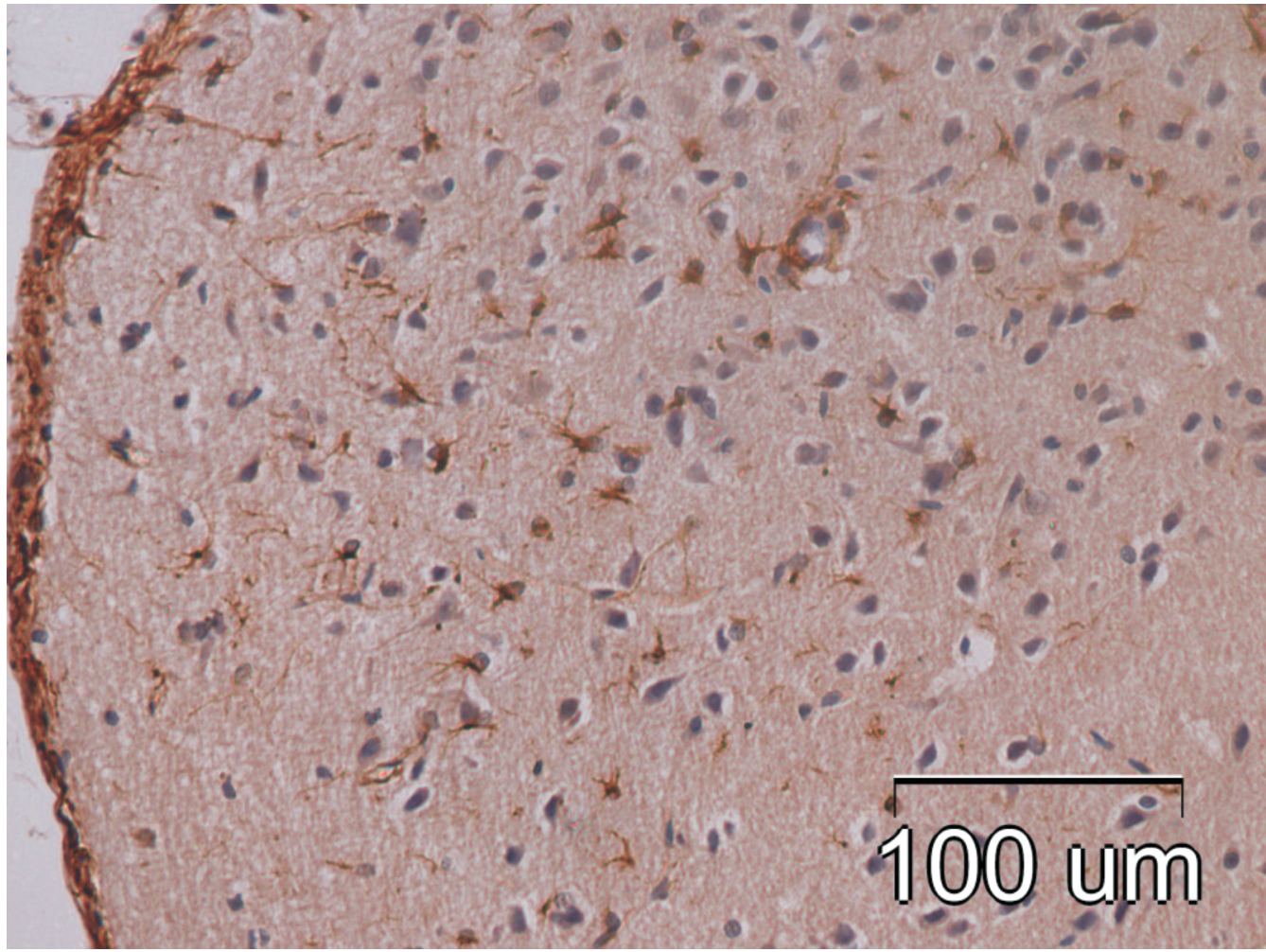

Fig. 3. Immunoreactivity of the GFAP-positive astrocytes in the ARC of E2 group.

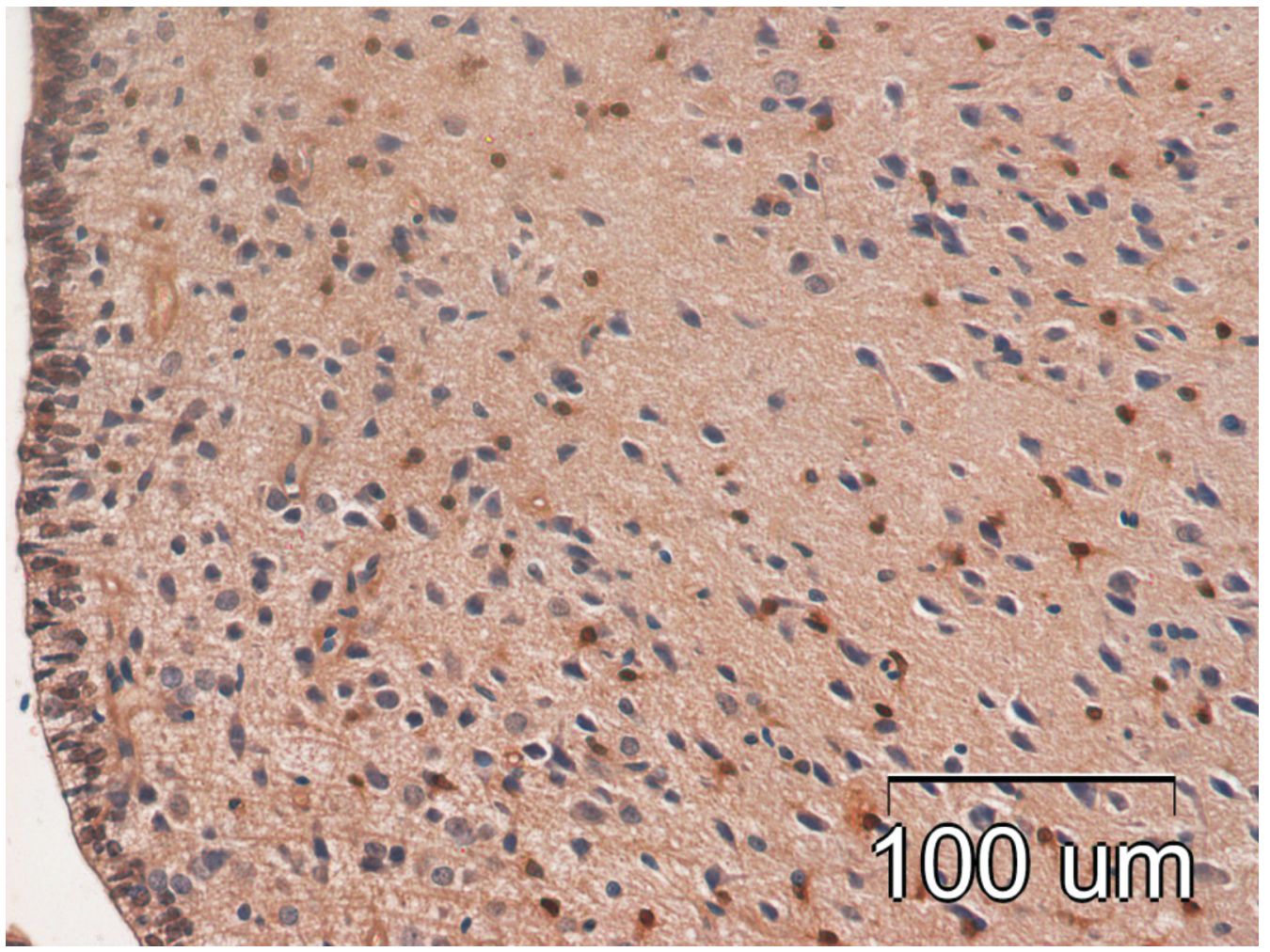

Fig. 4. Immunoreactivity of the S100ß-positive astrocytes in the ARC of C group. 


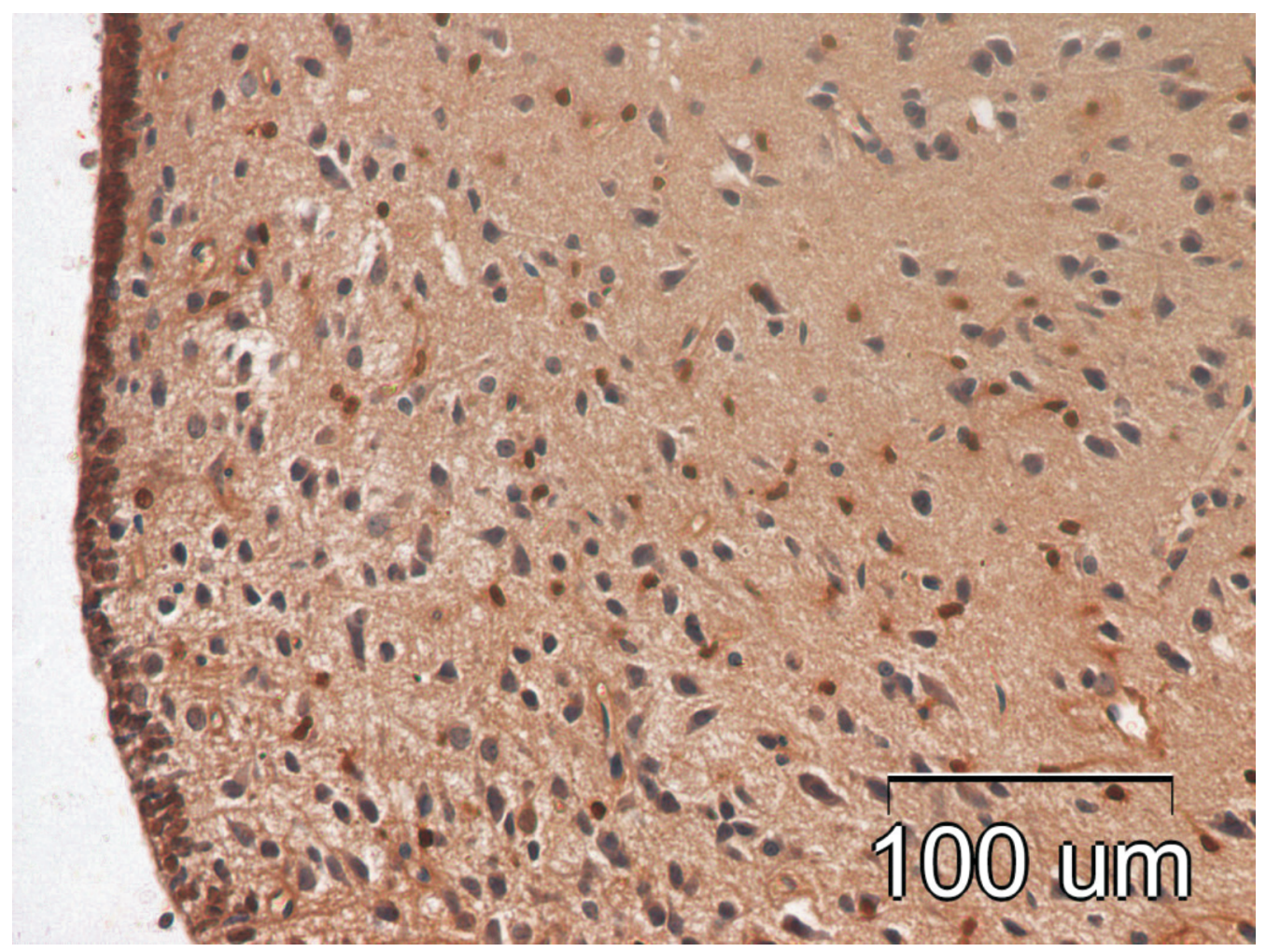

Fig. 5. Immunoreactivity of the S100ß-positive astrocytes in the ARC of E1 group.

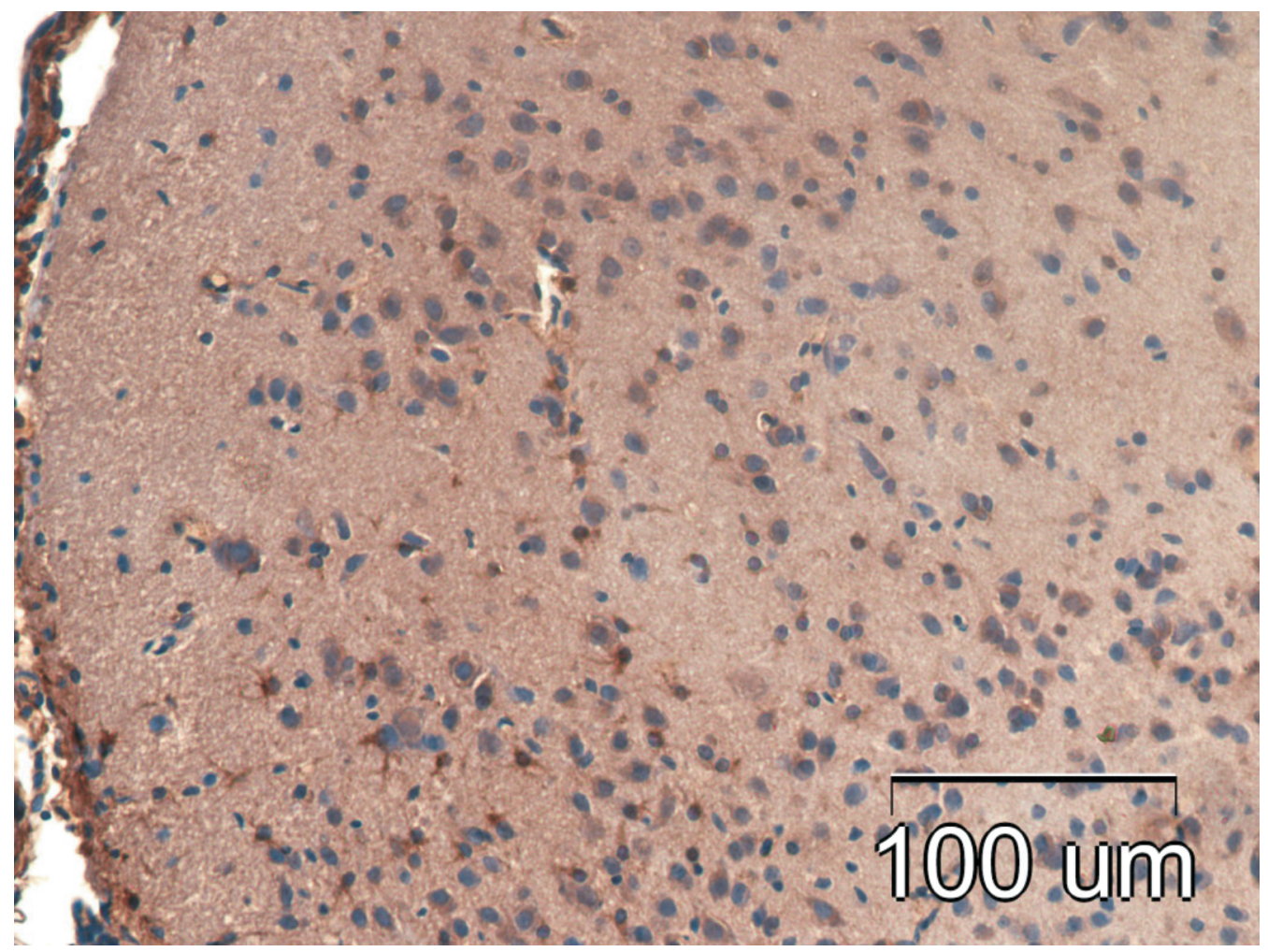

Fig. 6. Immunoreactivity of the S100ß-positive astrocytes in the ARC of E2 group. 


\section{Morphometric analyses of the GFAP-IR and S100/-IR astrocytes in ARC}

Morphometric and statistical analyses of the average density of the GFAP-positive cells in ARC of the E1 and E2 groups did not significantly differ in comparison with $\mathrm{C}$ animals (Fig. 7).

On the other hand, morphometric and statistical analyses of the average density of astrocytes with $S 100 \beta$ protein expression in E1 and E2 groups showed statistically significant differences in comparison with $\mathrm{C}$ animals. A statistically significant increase of S100 3 -immunopositive astrocytes was observed in ARC of the E1 group. On the other hand, a statistically significant decrease in the average astrocytic cell density was present in ARC of the experimental E2 group (Fig. 8).

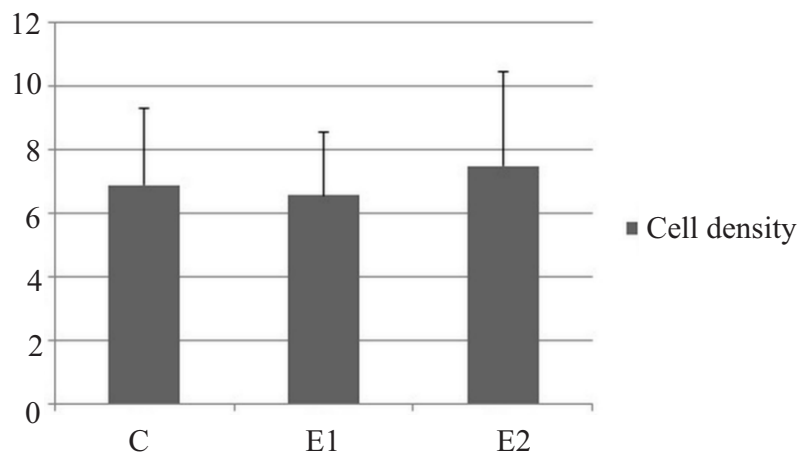

Fig. 7. Average density of GFAP-IR in ARC of the studied groups of rats (C, E1 and E2). Data shows mean density of GFAP-immunopositive cells in an area of $22.5 \times 10^{-3} \mathrm{~mm}^{2}$ with standard deviation bars.

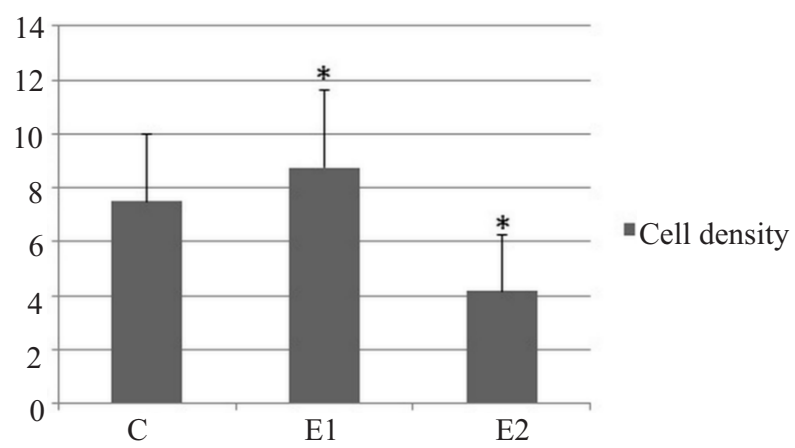

Fig. 8. Average density of S100 $\beta$-IR cells in ARC of the studied groups of rats (C, E1 and E2) Data shows mean density of S100 $\beta$-immunopositive cells in an area of 22.5 x $10^{-3} \mathrm{~mm}^{2}$ with standard deviation bars.

* statistically significant differences (Kruskal-Wallis, $\mathrm{p}<0.05$ ).

\section{Discussion}

Our studies revealed a slight influence of habanero pepper fruits, and thus of CAP, on the GFAP-IR cell density in ARC after 8-day and 4-week intragastric administration to rats (groups E1 and E2) in comparison with the control group (C). However, morphological alterations were observed in the E2 group. A few primary processes arose from one pole of the oval cell bodies. They divided into secondary, smaller branches. These changes may suggest the activation (hypertrophy) of astrocytes caused by 4 -week administration of habanero pepper fruits. We suggest that some immunohistochemically stained glial processes may be invisible due to a loss of GFAP in IFs. This may indicate an increase in mobility and prolongation of astrocytic processes to protect the neurons. Astrocytes in vivo and in vitro are capable of producing three proteins: GFAP, vimentin and nestin which are bound to IFs. Studies of primary astrocytic cultures revealed that the absence of GFAP and vimentin completely abolishes the production of IFs. Without IFs astrocytes expressed significant changes of shape. They were more oval with a few short processes. The results of these studies indicate that the IFs are directly involved in prolongation of the glial processes (Lepekhin et al. 2001). On the other hand, the presence of the GFAP stabilizes the cell shape and limits the plasticity of the glial processes. Therefore, the loss of the GFAP may increase the glial processes mobility and they may extend in the direction of synapses. In proper conditions the cells affect neuronal transmission. They uptake the excess of calcium ions and mainly glutamate (Glu) from the extracellular space. Glu enters the astrocytes by specific transporters: glutamate glial transporter 1 (GLT-1) and glial glutamate and aspartate transporter (GLAST) (Bélanger and Magistretti 2009). The absence of GFAP in Ifs may lead to intracellular glutamine synthesis from glutamate (Lepekhin et al. 2001). Our results suggest that the CAP present in the habanero pepper fruits may cause a reduction of the GFAP content in some glial processes in ARC, which in consequence may lead to alterations in the neuronal microenvironment, neuron-astrocyte interaction and neuronal nets plasticity. Different results were obtained in rats $75 \mathrm{~min}$ after subcutaneous administration of CAP. A significant increase in GFAP immunoreactivity and glutamine synthetase (GS) was demonstrated in ARC and ME astrocytes. The authors of this work suggest that these alterations affect a functional adaptation of astrocytes to cope with the CAP effect in order to maintain a proper microenvironment for neurons. GS activity induced by CAP indicates the increased level of Glu uptake by astrocytes from the intracellular space (Okere and Waterhouse 2004). Comparing our own results with other authors' results, we suggest that the astrocytes may respond differently depending on the route and time of CAP administration. For the first time, we studied the ex- 
pression of the $\mathrm{S} 100 \beta$ protein in astrocytes after intragastric administration of CAP contained in habanero pepper fruits. We demonstrated the time-dependent effect of these fruits on the density and morphology of astrocytes with S100 $\beta$ expression in ARC. The shorter time of administration of the fruits caused a statistically significant increase in glial cell density in the E1 group. This may be a consequence of intensified synthesis of the studied calcium binding protein caused by an influx of calcium ions into the astrocytes. Our studies suggest indirectly that regulatory mechanisms are activated in the astrocytes. S100 $\beta$ may increase the frequency of cell divisions, remodelling the cell cytoskeleton via IFs formation inhibition (Yashuda et al. 2004). One of the most basic features of astroglia reactivity is the synthesis of S100 $\beta$. This calcium binding protein reacts to changes of intracellular calcium ion levels and decreases their concentration. This buffer protein takes part in calcium-dependant cellular processes. It has been demonstrated that it blocks microtubule synthesis or that it stimulates the breakdown of gliofilaments formed by GFAP (Donato 2003). On the other hand, a longer time of habanero fruits administration, and thus higher levels of CAP in ARC of the E2 group, resulted in a statistically significant decrease of the density of astrocytes with $\mathrm{S} 100 \beta$ expression in comparison to group $\mathrm{C}$. This suggests that a release of $S 100 \beta$ from astrocytes to intracellular space may be increased and this autocrinally and paracrinally affects neurons and different types of glia. We observed the appearance of glial processes, which is the result of astrocytes reactivity (Donato 2003). Other authors revealed that in the hippocampus of an epileptic model an excitation of astrocytic Glu metabotropic receptors type II (mGluR3) results in $\mathrm{S} 100 \beta$ secretion from astrocytes and in consequence this leads to a decrease in the number of these glial cells with $\mathrm{S} 100 \beta$ expression. The protein also shows a neuroprotective effect and increases the survival of specific neuronal populations (Sakatani et al. 2008). Phenotypic astroglial alterations in ARC indicate that the $\mathrm{BBB}$ is permeable for CAP derived from habanero pepper fruits after intragastric administration to rats.

CAP is passively resorbed by about $50 \%$ in the stomach and then by about $25-40 \%$ in the small intestine in human and rats after oral administration. It enters the liver via the portal vein and is metabolized. However, it reaches the brain with blood in $<5 \%$ (O'Neill et al. 2012). Currently, it is believed that CAP passes BBB regardless of the route of administration. Intraperitoneal, subcutaneous, intracutaneous and oral administration affects the selective activation of TRPV1 receptor (Güler et al. 2012). Neurons and astrocytes in CNS contain TRPV1 receptor which is a cation channel for $\mathrm{Ca}^{2+}$. The activation of capsaicin receptor by the ligand leads to $\mathrm{Ca}^{2+}$ influx to the cells (O'Neil and Brown 2003, Doly et al. 2004, Olszewska 2010, Kopczyńska 2011, O’Neill et al. 2012). However, other authors suggest that only a certain subpopulation of astrocytes (i.e. in substantia nigra) express the TRPV1 receptor (Ho et al. 2012). The TRPV1 receptor activation caused by CAP may stimulate astrocytes to produce endogenous neurotrophic factors including ciliary neurotrophic factor (CNTF). This neurotrophin, released to intracellular space, stimulates CNTF receptor localised in the membrane of neurons. This factor prevents degeneration of dopaminergic neurons in substantia nigra and modulates neuronal functions in an animal Parkinson's disease model after intraperitoneal administration of CAP to female rats (Nam et al. 2015). Only a few studies conducted in 6-7-week-old Sprague-Dawley rats revealed that CAP in a fat rich diet significantly reduces NPY expression in hypothalamic nuclei neurons (including ARC), the amygdala and brain cortex. In consequence, it contributes to a reduction in food intake and weight loss (Park et al. 2004).

To sum up, our results demonstrated that the period of intragastric administration of CAP contained in habanero pepper fruits has no significant influence on the density of GFAP-IR astrocytes in $\mathrm{ARC}$ of the E1 and E2 groups of rats, whereas the density of S100ß-IR cells is dependent on the period of administration of the fruit. The shorter time of administration leads to an increase in the density of astrocytes with S100 $\beta$ expression in ARC (E1 group). Longer period of habanero peppers administration (E2 group) causes the decrease of the density of astrocytes. Morphological alterations of GFAP-IR and S100 $\beta$-IR glial cells were observed in the E2 group of rats after a longer period of habanero pepper fruit treatment. The obtained results indicate astroglia activation, which is probably a result of neuroprotection. The alterations we demonstrated may have an influence on astrocyte-neuron interactions to maintain proper neuronal activity. Further studies of astrocytes and neurons are necessary in order to demonstrate the impact of habanero pepper fruits on the nervous system.

\section{References}

Bélanger M, Magistretti PJ (2009) The role of astroglia in neuroprotection. Dialogues Clin Neurosci 11: 281-295.

Belousov AB, van den Pol AN (1997) Local synaptic release of glutamate from neurons in the rat hypothalamic arcuate nucleus. J Physiol 499: 747-761. 
Doly S, Fischer J, Salio C, Conrath M (2004) The vanilloid receptor-1 is expressed in rat spinal dorsal horn astrocytes. Neurosci Lett 357: 123-126.

Donato R (2003) Intracellular and extracellular roles of S100 proteins. Microsc Res Tech 60: 540-551.

Eng LF, Ghirnikar RS, Lee YL (2000) Glial fibrillary acidic protein: GFAP-thirty-one years (1969-2000). Neurochem Res 25: 1439-1451.

Güler AD, Rainwater A, Parker JG, Jones GL, Argilli E, Arenkiel BR, Ehlers MD, Bonci A, Zweifel LS, Palmiter RD (2012) Transient activation of specific neurons in mice by selective expression of the capsaicin receptor. Nat Commun 3: 746.

Ho KW, Ward NJ, Calkins DJ (2012) TRPV1: a stress response protein in the central nervous system. Am J Neurodegener Dis 1: 1-14.

Kopczyńska B (2011) Cannabinoids' influence on cardio-respiratory response in an animal model. Med Weter 67: 613-617.

Lepekhin EA, Eliasson C, Berthold CH, Berezin V, Bock E, Pekny M (2001) Intermediate filaments regulate astrocyte motility. J Neurochem 79: 617-625.

Monsereenusorn Y (1983) Subchronic toxicity studies of capsaicin and capsium in rats. Res Commun Chem Pathol Pharmacol 41: 95-110.

Montgomery DL (1994) Astrocytes: form, functions, and roles in disease. Vet Pathol 31: 145-167.

Nam JH, Park ES, Won SY, Lee YA, Kim KI, Jeong JY, Baek JY, Cho EJ, Jin M, Chung YC, Lee BD, Kim SH, Kim EG, Byun K, Lee B, Woo DH, Lee CJ, Kim SR, Bok E, Kim YS, Ahn TB, Ko HW, Brahmachari S, Pletinkova O, Troconso JC, Dawson VL, Dawson TM, Jin BK (2015) TRPV1 on astrocytes rescues nigral dopamine neurons in Parkinson's desease via CNTF. Brain 138: $3610-3622$.

Okere CO, Waterhouse BD (2004) Capsaicin increases GFAP and glutamine synthetase immunoreactivity in rat arcuate nucleus and median eminence. Neuroreport 15: $255-258$

Olszewska J (2010) Capsaicin - cure or poison? Kosmos 59: 133-139.
O’Neill J, Brock C, Olesen AE, Andresen T, Nilsson M, Dickenson AH (2012) Unravelling the mystery of capsaicin: a tool to understand and treat pain. Pharmacol Rev 64: 939-971.

O'Neil RG, Brown RC (2003) The vanilloid receptor family of calcium-permeable channels: molecular integrators of microenvironmental stimuli. News Physiol Sci 18: 226-231.

Park ES, Jo S, Yi SJ, Kim JS, Ee HS, Lee IS, Seo KM, Sung JK, Lee I, Yoon YS (2004) Effect of capsaicin on cholecystokinin and neuropeptide $\mathrm{Y}$ expressions in the brain of high-fat diet fed rats. J Vet Med Sci 66: 107-114.

Pieńko T (2013) Capsaicin - properties, applications today and future prospects. Biul Wydz Farm WUM 2: 11-17.

Sakatani S, Seto-Ohshima A, Shinohara Y, Yamamoto Y, Yamamoto H, Itohara S, Hirase H (2008) Neural-activity-dependent release of $\mathrm{S} 100 \beta$ from astrocytes enhances kainate-induced gamma oscillations in vivo. J Neurosci 28: 10928-10936.

Simone DA, Nolano M, Johnson T, Wendelschafer-Crabb G, Kennedy WR (1998) Intradermal injection of capsaicin in humans produces degeneration and subsequent reinnervation of epidermal nerve fibers: correlation with sensory function. J Neurosci 18: 8947-8959.

Sugimoto T, Xiao C, Ichikawa H (1998) Neonatal primary neuronal death induced by capsaicin and axotomy invovles an apoptotic mechanism. Brain Res 807: 147-154.

Surh YJ, Lee SS (1995) Capsaicin, a double edged sword: toxicity, metabolism, and chemopreventive potential. Life Sci 56: 1845-1855.

Tardy M, Le Prince G, Babajko S, Riol H, Fages C, Rolland $B$ (1993) GFAP gene expression in normal and reactive astrocytes. In: Fedoroff S, Juurlink BHJ, Doucette R (eds) Biology and pathology of astrocyte-neuron interactions. Plenum Press, New York, pp 153-161.

Tóth A, Boczhn J, Kedei N, Lizanecz E, Bagi Z, Papp Z, Edes I, Csiba L, Blumberg PM (2005) Expression and distribution of vanilloid receptor 1 (TRPV1) in the adult rat brain. Brain Res Mol Brain Res 135: 162-168.

Yasuda Y, Tateishi N, Shimoda T, Satoh S, Ogitani E, Fujita S (2004) Relationship between S100beta and GFAP expression in astrocytes during infarction and glial scar formation after mild transient ischemia. Brain Res 1021: 20-31. 